\title{
DEGENERATING 0 IN TRIANGULATED CATEGORIES
}

\author{
MANUEL SAORÍN AND ALEXANDER ZIMMERMANN
}

\begin{abstract}
In previous work, based on work of Zwara and Yoshino, we defined and studied degenerations of objects in triangulated categories analogous to degeneration of modules. In triangulated categories it is surprising that the zero object may degenerate. We study this systematically. In particular we show that the degeneration of the zero object actually induces all other degenerations by homotopy pullback, that degeneration of 0 is closely linked, but not equivalent, to having zero image in the Grothendieck group.
\end{abstract}

\section{INTRODUCTION}

Degeneration of modules were intensively studied by e.g. Gabriel [5], Huisgen-Zimmermann, Riedtmann 14, Zwara 22, 23, since at least 1974, and was highly successful in various constructions. Degeneration of modules is defined by the following setting. Let $k$ be an algebraically closed field, and let $A$ be a finite dimensional $k$-algebra. Then the $A$-module structures on the vector space $k^{d}$ form an affine algebraic variety $\bmod (A, d)$ on which $G L_{d}(k)$ acts by conjugation. Isomorphism classes correspond to orbits under this action and an $A$-module $M$ degenerates to $N$ if the point corresponding to $N$ belongs to the Zariski closure of the $G L_{d}(k)$-orbit of the point corresponding to $M$. We write $M \leq_{\operatorname{deg}} N$ in this case. Riedtmann and Zwara showed that $M \leq_{\operatorname{deg}} N$ if and only if there is an $A$-module $Z$ and a short exact sequence $0 \rightarrow Z \rightarrow Z \oplus M \rightarrow N \rightarrow 0$. The last relation is denoted by $M \leq_{\text {Zwara }} N$. In collaboration with Jensen and $\mathrm{Su}[\underline{8}$ the second named author started to study an analogous concept for derived categories with a geometrically inspired concept based on orbit closures, and then in [9] more generally for triangulated categories based on Zwara's characterisation replacing short exact sequences by distinguished triangles. This last relation is denoted by the symbol $\leq_{\Delta}$. Both concepts were highly successfully used in many places, cf e.g. 10, 11, 12, 3, 4, 6, 7, 21. Independently Yoshino 20, gave a scheme theoretic definition for degenerations in the (triangulated) stable category of maximal Cohen-Macaulay modules, and he highlighted that in $M \leq_{\Delta} N$ one should assume that the induced endomorphism on $Z$ should be nilpotent. We denote the relation by $\leq_{\Delta+\text { nil }}$ in this case. Yoshino's scheme theoretic approach was a model for us to give a more general geometric definition for degeneration, which was achieved in [16] by introducing a scheme theoretic degeneration $\leq_{\text {cdeg. }}$. We then showed that, in case $\mathcal{T}$ has split idempotents, $M \leq_{\text {cdeg }} N$ always implies $M \leq_{\Delta+\text { nil }} N$, for objects $M, N \in \mathcal{T}$, the converse being also true when $\mathcal{T}$ is the subcategory of compact objects of a compactly generated algebraic triangulated category. We gave the rather technical definition of $\leq_{\text {cdeg }}$ by means of an ambient triangulated category and a natural transformation $t$ of the identity functor of this category satisfying a certain number of axioms. The actual degeneration $M \leq_{\text {cdeg }} N$ is then given by an object $Q$ in this ambient category such that the cone of $t_{Q}$ is $N$, and such that $M$ and $Q$ become isomorphic when one inverts all morphisms $t_{X}$ for all objects $X$ in the ambient category in the Gabriel-Zisman sense. For more ample details we refer to Section 1

A striking phenomenon is that, unlike in the module case, in triangulated categories $\mathcal{T}$ one may have non zero objects $M$ with $0 \leq{ }_{\Delta+\text { nil }} M$. In the present paper we study this phenomenon systematically.

As a first result we show that $0 \leq_{\mathrm{cdeg}} M$ is given by objects $Q$ so that the identity transformation $t$ of the degeneration data has the property that $t_{Q}$ is nilpotent.

Second, we show in the present paper that if $M \leq_{\Delta} N$ via a distinguished triangle

$$
Z \stackrel{\left(\begin{array}{l}
\alpha \\
\beta
\end{array}\right)}{\longrightarrow} Z \oplus M \stackrel{(\gamma, \delta)}{\longrightarrow} N \longrightarrow Z[1]
$$


then $0 \leq_{\Delta} \operatorname{cone}(\alpha)$ and $0 \leq_{\Delta} \operatorname{cone}(\delta)$, and if $0 \leq_{\Delta} X$ via a distinguished triangle

$$
Z \stackrel{\alpha}{\longrightarrow} Z \longrightarrow X \longrightarrow Z[1]
$$

then for any morphism $M \longrightarrow Z$ the homotopy pullback $N$ along $M \longrightarrow Z \stackrel{\alpha}{\longleftarrow} Z$ gives a degen-

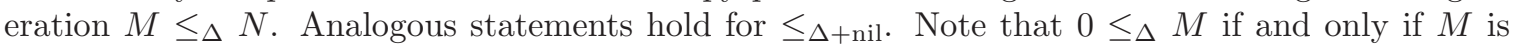
the cone of an endomorphism $v$ of some object $Z$, and $0 \leq_{\Delta+\text { nil }} M$ if and only if $v$ is in addition nilpotent. Hence, degeneration of 0 is very intrinsic in degenerations of triangulated categories. It is not possible to get rid of this phenomenon, when one wants to study degenerations. Therefore, our observation interprets degeneration as some sort of deformation along a (nilpotent) endomorphism of some parameter space $Z$ via a homotopy pullback construction.

Third, it is quite clear that $0 \leq_{\Delta} N$ implies that $N$ has vanishing image in the Grothendieck group of the triangulated category. We characterise the objects with image being the zero object in the Grothendieck group by showing that this condition is equivalent to the fact that $M \leq_{\Delta}$ $\bigoplus_{i=1}^{r}\left(X_{i} \oplus X_{i}\left[t_{i}\right]\right)$ for pairwise different odd integers $t_{i}$, and objects $X_{i}$ of $\mathcal{T}$. This indicates already that the objects $X \oplus X[t]$, with $t$ odd, are very intimately linked to degeneration phenomena. When $X$ runs through a generating set of the triangulated category, we show that the triangulated category generated by these objects coincides with the full triangulated subcategory $\mathcal{T}^{0}$ of $\mathcal{T}$ consisting of objects with image 0 in the Grothendieck group. We further show that the triangulated subcategory generated by objects which are degenerations of 0 coincides with $\mathcal{T}^{0}$. We give a class of examples of a triangulated category $\mathcal{T}$, and an object $X$ in $\mathcal{T}$ such that $[X]=0$ in $K_{0}(\mathcal{T})$, but $0 \npreceq \Delta+$ nil $X$, where $\preceq_{\Delta+\text { nil }}$ is the transitive hull of the relation $\leq_{\Delta+\text { nil }}$. It is not hard to get another class of examples where $[X]=0$ in $K_{0}(\mathcal{T})$ but where $0 \not \not_{\Delta} X$.

The paper is organised as follows. In Section 1 we recall the necessary concepts on the various types of degeneration. In Section 2 we study $\leq_{\text {cdeg }}$ and show that $0 \leq_{\text {cdeg }} M$ if and only if $t_{Q}$ is nilpotent for the corresponding degeneration data. In Section 3 we show that triangle degeneration of zero is always present in triangle degenerations in triangulated categories. Indeed all triangle degenerations are obtained from degenerations of zero via homotopy cartesian squares. In Section 4 we study the image of triangle degenerations of 0 in the Grothendieck group.

\section{Review on Degenerations in Triangulated Categories}

We have different degeneration concepts. The first one, the triangle degeneration, is a triangular category analogue of Zwara's definition of degeneration in the case of module categories. Zwara says 22, 23. that for a $k$-algebra $A$ an $A$-module $M$ degenerates to an $A$-module $N$ if and only if there is an $A$-module $Z$ and a short exact sequence $0 \rightarrow Z \rightarrow Z \oplus M \rightarrow N \rightarrow 0$. Yoshino 20] highlighted the importance of assuming that the induced endomorphism of $Z$ should be nilpotent. In case of a category where Fitting's lemma holds we can always assume this fact.

Definition 1. 9, 16, Let $K$ be a commutative ring and let $\mathcal{T}$ be a $K$-linear triangulated category. Then for two objects $M$ and $N$ in $\mathcal{T}$ we get $M \leq_{\Delta} N$, accasionally written $M \leq_{\Delta \text {, left }} N$, if and only if there is $Z$ and a distinguished triangle

$$
Z \stackrel{\left(\begin{array}{l}
v \\
u
\end{array}\right)}{\longrightarrow} Z \oplus M \longrightarrow N \longrightarrow Z[1]
$$

We say that $M \leq_{\Delta+\text { nil }} N$, or occasionally $M \leq_{\Delta+\text { nil, left }} N$, if and only if there is such a distinguished triangle with $v$ is nilpotent.

Note that by [17 $M \leq_{\Delta+\text { nil }} N$ implies that there is an object $Z^{\prime}$ and a distinguished triangle

$$
N \longrightarrow Z^{\prime} \oplus M \stackrel{\left(v^{\prime}, u^{\prime}\right)}{\longrightarrow} Z^{\prime} \longrightarrow N[1] .
$$

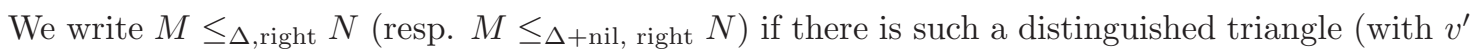
nilpotent). Note that $M \leq_{\Delta \text {, left }} N$ (resp. $\left.M \leq_{\Delta+\text { nil,left }} N\right)$ in $\mathcal{T}$ if and only if $M \leq_{\Delta \text {,right }} N$ (resp. $\left.M \leq_{\Delta+\text { nil,right }} N\right)$ in the opposite category $\mathcal{T}^{o p}$. So categorical duality applies and results about

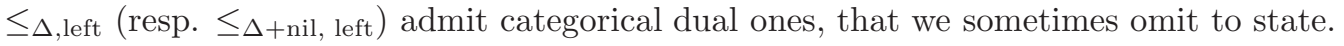

If $\mathcal{T}$ has split idempotents and artinian endomorphism rings of objects, or if $\mathcal{T}$ is the category of compact objects in a compactly generated algebraic triangulated category, then $M \leq_{\Delta+\text { nil, right }} N$ if 


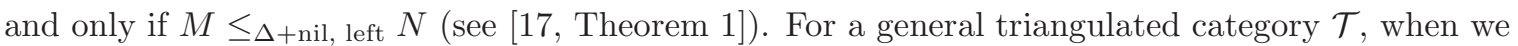
write $\leq_{\Delta}\left(\right.$ resp. $\left.\leq_{\Delta+\text { nil }}\right)$ we will mean $\leq_{\Delta \text {,left }}\left(\right.$ resp. $\left.\leq_{\Delta+\text { nil, left }}\right)$

Corollary 2. Let $K$ be a commutative ring and let $\mathcal{T}$ be a $K$-linear triangulated category. Then $M \leq_{\Delta} N$ implies that $[M]=[N]$, where $[X]$ denotes the image of $X$ in $K_{0}(\mathcal{T})$.

Indeed, this is a direct consequence of the fact that there is a distinguished triangle

$$
Z \stackrel{\left(\begin{array}{l}
v \\
u
\end{array}\right)}{\longrightarrow} Z \oplus M \longrightarrow N \longrightarrow Z[1]
$$

The distinguished triangle shows $[Z]+[N]-[Z \oplus M]=0$ in the Grothendieck group, and therefore $[M]=[N]$.

A second concept of degeneration, motivated by Yoshino's work, is given by the following definition.

Definition 3. [16] Let $K$ be a commutative ring and let $\mathcal{C}_{K}^{\circ}$ be a $K$-linear triangulated category with split idempotents.

A degeneration data for $\mathcal{C}_{K}^{\circ}$ is given by

- a triangulated category $\mathcal{C}_{K}$ with split idempotents and a fully faithful embedding $\mathcal{C}_{K}^{\circ} \longrightarrow \mathcal{C}_{K}$,

- a triangulated category $\mathcal{C}_{V}$ with split idempotents and a full triangulated subcategory $\mathcal{C}_{V}^{\circ}$,

- triangulated functors $\uparrow V_{K}: \mathcal{C}_{K} \longrightarrow \mathcal{C}_{V}$, which we write after the arguments, and $\Phi: \mathcal{C}_{V}^{\circ} \rightarrow \mathcal{C}_{K}$, so that $\left(\mathcal{C}_{K}^{\circ}\right) \uparrow_{K}^{V} \subseteq \mathcal{C}_{V}^{\circ}$, when we view $\mathcal{C}_{K}^{\circ}$ as a full subcategory of $\mathcal{C}_{K}$,

- a natural transformation $\operatorname{id}_{\mathcal{C}_{V}} \stackrel{t}{\longrightarrow} \operatorname{id}_{\mathcal{C}_{V}}$ of triangulated functors such that

- for each object $M$ of $\mathcal{C}_{K}^{\circ}$ the morphism $\Phi\left(M \uparrow_{K}^{V}\right) \stackrel{\Phi\left(t_{M \uparrow K}\right)}{\longrightarrow} \Phi\left(M \uparrow_{K}^{V}\right)$ is a split monomorphism in $\mathcal{C}_{K}$ with cone $M$.

Degeneration is then given by the following concept.

Definition 4. [16] Given two objects $M$ and $N$ of $\mathcal{C}_{K}^{\circ}$ we say that $M$ degenerates to $N$ in the categorical sense if there is a degeneration data for $\mathcal{C}_{K}^{\circ}$ and an object $Q$ of $\mathcal{C}_{V}^{\circ}$ such that

$$
p(Q) \simeq p(M \uparrow \underset{K}{V}) \text { in } \mathcal{C}_{V}^{\circ}\left[t^{-1}\right] \text { and } \Phi\left(\operatorname{cone}\left(t_{Q}\right)\right) \simeq N,
$$

where $\mathcal{C}_{V}^{\circ}\left[t^{-1}\right]$ is the Gabriel-Zisman localisation at the endomorphisms $t_{X}$ for all objects $X$ of $\mathcal{C}_{V}^{\circ}$, and where $p: \mathcal{C}_{V}^{\circ} \longrightarrow \mathcal{C}_{V}^{\circ}\left[t^{-1}\right]$ is the canonical functor. In this case we write $M \leq_{\text {cdeg }} N$.

Remark 5. We note that this concept is a generalisation to general triangulated categories of a definition given by Yoshino 20] for the case of stable categories of maximal Cohen-Macaulay modules over a local Gorenstein algebra.

We get the following connection on these two concepts $\leq_{\Delta+\text { nil }}$ and $\leq_{\text {cdeg. }}$.

Theorem 6. [16] Let $\mathcal{T}$ be a $K$-linear triangulated category with split idempotents. Then

$$
M \leq_{\text {cdeg }} N \Rightarrow M \leq_{\Delta+\text { nil }} N
$$

and if $\mathcal{T}$ is the category of compact objects in a compactly generated algebraic triangulated category then

$$
M \leq_{\text {cdeg }} N \Leftrightarrow M \leq_{\Delta+\text { nil }} N .
$$

Corollary 7. Let $\mathcal{T}$ be a K-linear triangulated category with split idempotents. Then

$$
M \leq_{\mathrm{cdeg}} N \Rightarrow[M]=[N]
$$

in $K_{0}(\mathcal{T})$.

Indeed, this is Theorem 6 in connection with Corollary 2

Let $\mathcal{T}$ be a skeletally small triangulated category. In [9] it is studied when $\leq_{\Delta}$ is a partial order on the isomorphism classes of objects in $\mathcal{T}$, and in particular when $\leq_{\Delta}$ is a transitive relation. In general this is not the case. However the following was proved there.

Proposition 8. 9, Proposition 2] Let $\mathcal{T}$ be a triangulated category with split idempotents such that the endomorphism ring of each object in $\mathcal{T}$ is artinian. Then $\leq_{\Delta+\text { nil }}$ coincides with $\leq_{\Delta}$ and both relations are transitive and reflexive on the set of isomorphism classes of objects in $\mathcal{T}$. 


\section{SCheme theOREtic Degeneration of ZERO}

2.1. Torsion Degeneration Data. Recall that in 19] Yoshino defines as well the scheme theoretic definition of a degeneration of modules. Let $A$ be a $k$-algebra for some field $k$. Yoshino says that the $A$-module $M$ degenerates to the $A$-module $N$ along a discrete valuation ring if there is a discrete valuation $k$-algebra $V$ with uniformiser $t$ and a finitely generated $A \otimes_{k} V$-module $Q$ which is flat as $V$-module, such that $Q / t Q \simeq N$, and such that $Q\left[t^{-1}\right] \simeq M \otimes_{k} V\left[t^{-1}\right]$. In [20] a similar setting was used for degeneration in a triangulated (stable) category, and there the condition on $Q$ to be flat over $V$ is missing. We now explain why this is the case.

Proposition 9. Let $\mathcal{C}_{K}^{\circ}$ be a $K$-linear triangulated category with split idempotents. Then $0 \leq_{\mathrm{cdeg}} N$ if and only if there is a degeneration data $\left(\mathcal{C}_{K}^{\circ}, \mathcal{C}_{K}, \mathcal{C}_{V}^{\circ}, \mathcal{C}_{V}, \uparrow V, \Phi, t\right)$ and $Q$ an object in $\mathcal{C}_{V}^{\circ}$ such that $t_{Q}$ is nilpotent with

$$
\operatorname{cone}\left(\Phi\left(t_{Q}\right)\right) \simeq \Phi\left(\operatorname{cone}\left(t_{Q}\right)\right) \simeq N \text { and } \Phi(Q) \in \mathcal{C}_{K}^{\circ}
$$

Proof. Suppose we have a degeneration data and by [16, Lemma 7] $Q$ an object in $\mathcal{C}_{V}^{\circ}$ so that $t_{Q}$ is nilpotent with

$$
\operatorname{cone}\left(\Phi\left(t_{Q}\right)\right) \simeq \Phi\left(\operatorname{cone}\left(t_{Q}\right)\right)=: N \in \mathcal{C}_{K}^{\circ} .
$$

Then $p(Q) \simeq 0$ since $t_{Q}$ is nilpotent. Hence, by definition $0 \leq_{c d e g} N$.

If $0 \leq_{\text {cdeg }} N$, then there is a degeneration data and an object $Q$ in $\mathcal{C}_{V}^{\circ}$ with nilpotent $t_{Q}$ and $\Phi\left(\operatorname{cone}\left(t_{Q}\right)\right)=N \in \mathcal{C}_{K}^{\circ}$. Note that the proof of the first part of Theorem 6 gives that actually $\Phi($ cone $(f))$ is the object named $Z$, where $f: M \uparrow \uparrow_{K} \longrightarrow Q$ is a morphism such that $p(f)$ is an isomorphism. In case $M=0$, then $\operatorname{cone}(f)=Q$, and hence $\Phi(Q)=Z \in \mathcal{C}_{K}^{\circ}$.

Remark 10. We just proved that when we want to consider degenerations $M \leq_{\text {cdeg }} N$ without direct factor $0 \leq_{\mathrm{cdeg}} X$, then it is necessary and sufficient to add as an additional condition that $Q$ does not have any non zero direct factor $Q^{\prime}$ with $\Phi\left(Q^{\prime}\right) \in \mathcal{C}_{K}^{\circ}$ such that $t_{Q^{\prime}}$ is nilpotent.

\subsection{Triangle degeneration of zero.}

Lemma 11. Let $\mathcal{T}$ be a triangulated category with split idempotents. Then $0 \leq_{\Delta} N$ (resp. $0 \leq_{\Delta+\text { nil }}$ $N$ ) is equivalent to $N=\operatorname{cone}(v)$ for some (resp. nilpotent) endomorphism $v$ of an object $Z$. Moreover, in this case $N \leq_{\Delta+\text { nil }} Z \oplus Z[1]$.

Proof. If $\mathcal{T}$ is a $K$-linear triangulated category with split idempotents. Then $0 \leq_{\Delta} N$ (resp. $0 \leq_{\Delta+\text { nil }}$ $N$ ) is equivalent to the existence of an object $Z$ of $\mathcal{T}$ and a (resp. nilpotent) endomorphism $v$ of $Z$ such that $N=\operatorname{cone}(v)$. In other words there is a distinguished triangle

$$
Z \stackrel{v}{\longrightarrow} Z \stackrel{\epsilon}{\longrightarrow} N \stackrel{\delta}{\longrightarrow} Z[1]
$$

Then, since

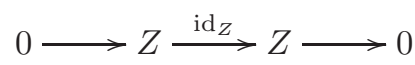

is a distinguished triangle, also

$$
Z \oplus Z[-1] \stackrel{\left(\begin{array}{ll}
v & 0
\end{array}\right)}{\longrightarrow} Z \stackrel{\left(\begin{array}{l}
\epsilon \\
0
\end{array}\right)}{\longrightarrow} N \oplus Z \stackrel{\left(\begin{array}{cc}
\delta & 0 \\
0 & \mathrm{id}_{Z}
\end{array}\right)}{\longrightarrow} Z[1] \oplus Z
$$

is a distinguished triangle. Shift to the left gives a distinguished triangle

$$
Z \stackrel{\left(\begin{array}{l}
\epsilon \\
0
\end{array}\right)}{\longrightarrow} N \oplus Z \stackrel{\left(\begin{array}{cc}
\delta & 0 \\
0 & \mathrm{id} z
\end{array}\right)}{\longrightarrow} Z[1] \oplus Z \stackrel{\left(\begin{array}{ll}
v & 0
\end{array}\right)}{\longrightarrow} Z[1]
$$

Now, 0 is clearly nilpotent, and hence $\left.N \leq_{\Delta+\text { nil }} Z \oplus Z[1]\right)$.

Theorem 12. Let $\mathcal{T}$ be a triangulated $k$-category. The following assertions hold:

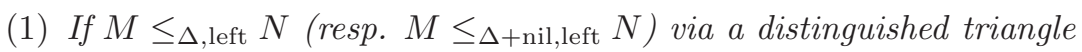

$$
Z \stackrel{\left(\begin{array}{l}
u \\
v
\end{array}\right)}{\longrightarrow} M \oplus Z \stackrel{\left(\begin{array}{ll}
\pi & \tau
\end{array}\right)}{\longrightarrow} N \stackrel{\mu}{\longrightarrow} Z[1]
$$




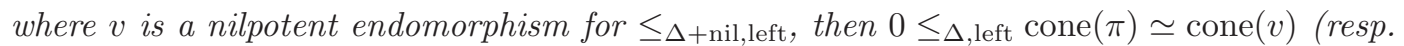
$\left.0 \leq_{\Delta+\text { nil, } \text { left }} \operatorname{cone}(\pi) \simeq \operatorname{cone}(v)\right)$

(2) If $M \leq_{\Delta \text {,right }} N$ (resp. $M \leq_{\Delta+\text { nil,right }} N$ ) via a distinguished triangle

$$
N \stackrel{\left(\begin{array}{c}
\sigma \\
\lambda
\end{array}\right)}{\longrightarrow} M \oplus Z^{\prime} \stackrel{\left(\begin{array}{ll}
u^{\prime} & v^{\prime}
\end{array}\right)}{\longrightarrow} Z^{\prime} \stackrel{\mu^{\prime}}{\longrightarrow} N[1],
$$

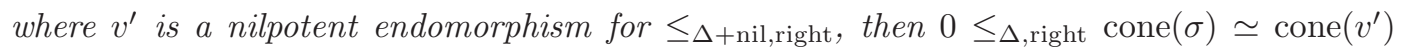

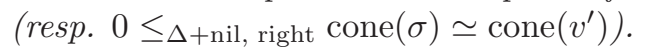

On the other hand, if $C$ is the cone of the (resp. nilpotent) endomorphism $v$ of $Z$, then for every

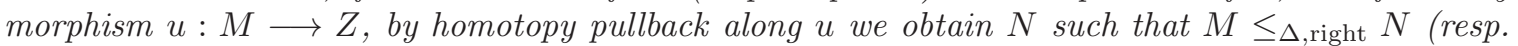

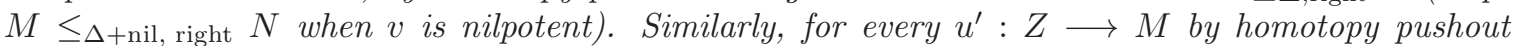

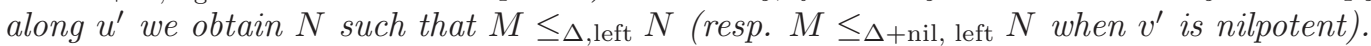

Proof. Assertion 2 is the categorical dual of assertion 1. So we just prove this last assertion. Consider the distinguished triangle

$$
Z \stackrel{\left(\begin{array}{l}
u \\
v
\end{array}\right)}{\longrightarrow} M \oplus Z \stackrel{\left(\begin{array}{ll}
\pi & \tau
\end{array}\right)}{\longrightarrow} N \longrightarrow Z[1]
$$

By definition, the square

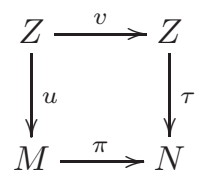

is homotopy cartesian. We then complete the horizontal maps to distinguished triangles to get a morphism between triangles.

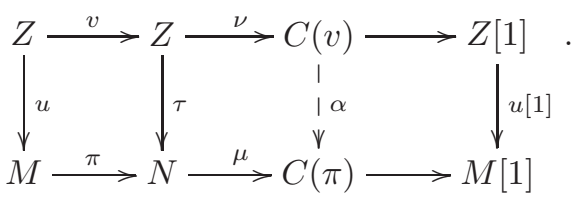

We may choose $\alpha$ to be an isomorphism and, by the upper row of the diagram, we conclude that

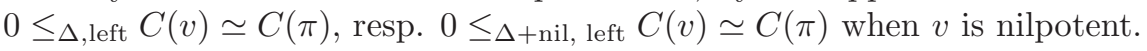

For the last statement, we just prove one half of it since the other half is dual. Let us consider the distinguished triangle

$$
Z \stackrel{v}{\longrightarrow} Z \longrightarrow C \longrightarrow Z[1]
$$

For any morphism $u: M \longrightarrow Z$ we may complete the diagram

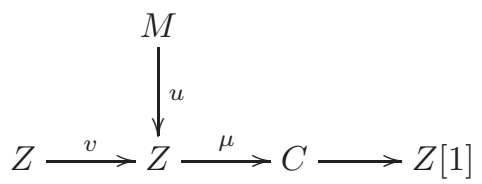

to

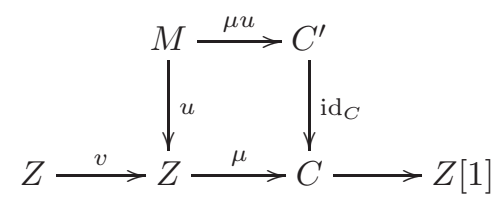

By the dual of Neeman [13, Lemma 1.4.3] there is an object $N$ and a morphism $v$ such that

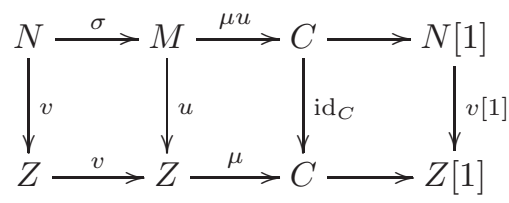


is a morphism of distinguished triangles such that

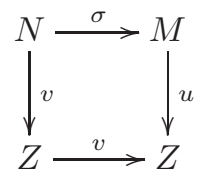

is a homotopy cartesian square. By definition this gives a distinguished triangle

$$
N \longrightarrow M \oplus Z \longrightarrow Z \longrightarrow N[1]
$$

Hence $M \leq_{\Delta \text {,right }} N$, (resp. $M \leq_{\Delta+\text { nil, right }} N$ when $v$ is nilpotent).

Definition 13. Let $\mathcal{T}$ be a triangulated category, let $M$ and $Z$ be objects in $\mathcal{T}$ and let $v$ be an endomorphism of $Z$. For $u \in \operatorname{Hom}_{\mathcal{T}}(M, Z)$ we denote by $(\operatorname{Deg}(u, v), r, s)$, or just $\operatorname{Deg}(u, v)$ for short, the homotopy pullback

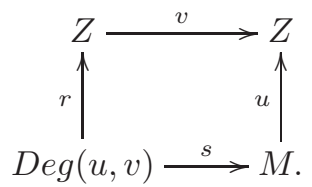

Dually, for $u^{\prime} \in \operatorname{Hom}_{\mathcal{T}}(Z, M)$ we denote by $\left(\operatorname{Ged}\left(u^{\prime}, v\right), r, s\right)$, or just $G e d\left(u^{\prime}, v\right)$ for short, the homotopy pushout

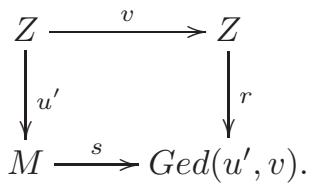

We then have

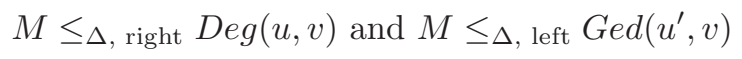

and if $v$ is in addition nilpotent, then

$$
M \leq_{\Delta+\text { nil, right }} \operatorname{Deg}(u, v) \text { and } M \leq_{\Delta+\text { nil, left }} \operatorname{Ged}\left(u^{\prime}, v\right) .
$$

Remark 14. Suppose $\mathcal{T}$ has split idempotents and either endomorphism rings of objects in $\mathcal{T}$ are artinian or else $\mathcal{T}$ is the category of compact objects in an algebraic compactly generated triangulated category. Then [17] shows that

- for each object $Z$, nilpotent endomorphism $v$ of $Z$ and morphism $M \stackrel{u}{\longrightarrow} Z$ there is an object $Z^{\prime}$, a nilpotent endomorphism $v^{\prime}$ of $Z^{\prime}$ and a morphism $Z^{\prime} \stackrel{u^{\prime}}{\longrightarrow} M$ with $\operatorname{Ged}\left(u^{\prime}, v^{\prime}\right) \simeq$ $\operatorname{Deg}(u, v)$,

- and for each object $Z^{\prime}$, nilpotent endomorphism $v^{\prime}$ of $Z^{\prime}$ and morphism $Z^{\prime} \stackrel{u^{\prime}}{\longrightarrow} M$ there is an object $Z$, a nilpotent endomorphism $v$ of $Z$ and a morphism $M \stackrel{u}{\longrightarrow} Z$ with $\operatorname{Ged}\left(u^{\prime}, v^{\prime}\right) \simeq$ $\operatorname{Deg}(u, v)$.

Example 15. Consider a finite dimensional $k$-algebra $A$ over an algebraically closed field $k$. Let $M$ and $N$ be two finite dimensional $A$-modules, and suppose $M \leq_{\operatorname{deg}} N$. Then by the Zwara-Riedtmann theorem 23] there is a finite dimensional $A$-module $Z$ and a short exact sequence

$$
0 \longrightarrow Z \stackrel{\left(\begin{array}{l}
v \\
u
\end{array}\right)}{\longrightarrow} Z \oplus M \stackrel{(\tau, \pi)}{\longrightarrow} N \longrightarrow 0 .
$$

A trivial case is when there is a short exact sequence

$$
0 \longrightarrow N_{1} \stackrel{\iota}{\longrightarrow} M \stackrel{\rho}{\longrightarrow} N_{2} \longrightarrow 0 \text {. }
$$

Taking $Z=N_{1}$ the short exact sequence

$$
0 \longrightarrow N_{1} \stackrel{\left(\begin{array}{l}
\iota \\
0
\end{array}\right)}{\longrightarrow} M \oplus N_{1} \stackrel{\left(\begin{array}{cc}
\rho & 0 \\
0 & \mathrm{id}_{N_{1}}
\end{array}\right)}{\longrightarrow} N_{2} \oplus N_{1} \longrightarrow 0
$$


is a Zwara-Riedtmann sequence. In particular, in this case $v=0$. Since $A-\bmod \hookrightarrow \mathcal{D}^{b}(A)$, by mapping a module to the corresponding stalk complex in degree 0 , we can consider the Zwara exact sequence as three terms of a distinguished triangle. By Theorem 12 the map $\pi$ in the general setting yields a degeneration $0 \leq_{\Delta+\text { nil }} \operatorname{cone}(\pi)$ in $\mathcal{D}^{b}(A)$. Of course there is no degeneration of 0 of modules. Hence, this phenomenon is a purely triangulated one. What does this give in this particular situation? Actually, for a general $\pi$, we get

$$
\operatorname{cone}(\pi)=(\ldots \longrightarrow 0 \longrightarrow M \stackrel{\pi}{\longrightarrow} N \longrightarrow 0 \longrightarrow \ldots)
$$

is the two term complex concentrated in degrees -1 and 0 . In the special situation of the short exact sequence

$$
0 \longrightarrow N_{1} \stackrel{\iota}{\longrightarrow} M \stackrel{\rho}{\longrightarrow} N_{2} \longrightarrow 0
$$

we see that $v=0$ and

$$
\operatorname{cone}(\pi)=\operatorname{cone}\left(\begin{array}{cc}
\rho & 0
\end{array}\right) \simeq\left(\ldots \rightarrow M \stackrel{\rho}{\rightarrow} N_{2} \rightarrow 0 \ldots\right) \oplus N_{1} \simeq \operatorname{ker}(\rho)[1] \oplus N_{1} \simeq N_{1}[1] \oplus N_{1}
$$

since $\rho$ is surjective, and hence the two term complex given by $\rho$ is isomorphic, in the derived category, to the kernel of $\rho$. This is coherent with the computation of the degeneration given by the zero map on $Z=N_{1}$.

\section{Triangle Degeneration as homotopy CARTESIAN SQUARE: SOME CONSEQUENCES}

3.1. The case of a single degeneration object. In view of Theorem 12 we consider degeneration as homotopy cartesian squares.

Lemma 16. Let $\mathcal{T}$ be a triangulated category, let $Z \stackrel{w}{\longrightarrow} M \stackrel{u}{\longrightarrow} Z$ be morphisms in $\mathcal{T}$ and let $\nu_{1}, \nu_{2}: Z \longrightarrow Z$ be endomorphisms. Denote $\operatorname{Deg}\left(u, \nu_{1}\right)=\left(\operatorname{Deg}\left(u, \nu_{1}\right), r_{1}, s_{1}\right)$ and $\operatorname{Ged}\left(w, \nu_{2}\right)=$ $\left(\operatorname{Ged}\left(w, \nu_{2}\right), r_{2}, s_{2}\right)$ for short. The following assertions hold:

(1) $\operatorname{Deg}\left(u, \nu_{1}\right) \leq_{\Delta \text {,right }} \operatorname{Deg}\left(u, \nu_{1} \nu_{2}\right)$ as well as $\operatorname{Deg}\left(u, \nu_{1} \nu_{2}\right)=\operatorname{Deg}\left(r_{1}, \nu_{2}\right)$. When $\nu_{2}$ is nilpotent, we can replace $\leq_{\Delta \text {,right }}$ by $\leq_{\Delta+\text { nil,right }}$.

(2) $\operatorname{Ged}\left(w, \nu_{2}\right) \leq_{\Delta \text {,left }} \operatorname{Ged}\left(w, \nu_{1} \nu_{2}\right)$ as well as $\operatorname{Ged}\left(u, \nu_{1} \nu_{2}\right)=\operatorname{Ged}\left(r_{2}, \nu_{1}\right)$. When $\nu_{1}$ is nilpotent, we can replace $\leq_{\Delta \text {, left }}$ by $\leq_{\Delta+\text { nil,left }}$.

Proof. Assertion 1 follows from assertion 2 by categorical duality, so we just prove assertion 2 . Let us consider the following diagram, where the upper inner and the outer squares are homotopy cartesian

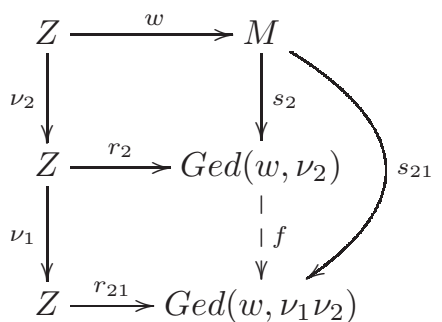

There is then a dotted morphism $f: \operatorname{Ged}\left(w, \nu_{2}\right) \longrightarrow \operatorname{Ged}\left(w, \nu_{1} \nu_{2}\right)$ completing commutatively the diagram (see [13, page 54]). But, by [17, Lemma 9], we can choose $f$ so that the square

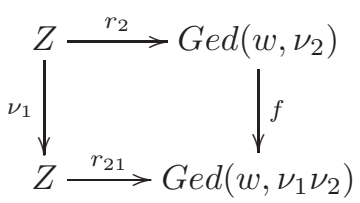

is homotopy cartesian. Therefore we have $\operatorname{Ged}\left(w, \nu_{2}\right) \leq_{\Delta \text {, left }} \operatorname{Ged}\left(w, \nu_{1} \nu_{2}\right)$ as well as $\operatorname{Ged}\left(u, \nu_{1} \nu_{2}\right)=$

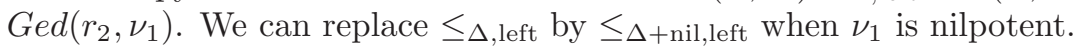


3.2. The case of two degeneration objects. In this subsection we show that a degeneration $M \leq_{\Delta \text {,right }} N$ obtained by two different triangles, but with the same morphism $N \longrightarrow M$, naturally yields another degeneration. Concretely:

Proposition 17. Let $\mathcal{T}$ be a triangulated category and let $M \leq_{\Delta \text {,right }} N$ (resp. $\left.M \leq_{\Delta+\text { nil,right }} N\right)$ be a degeneration obtained by the following homotopy cartesian squares

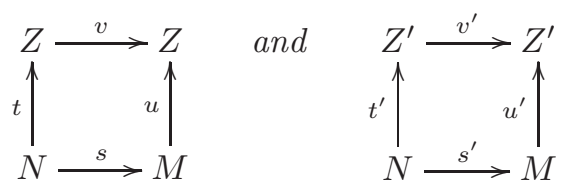

where $v$ and $v^{\prime}$ are assumed to be nilpotent endomorphism in the $\leq_{\Delta+\text { nil,right-case, and suppose that }}$

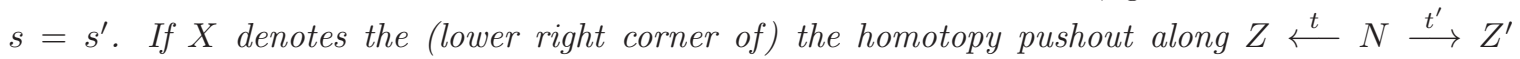

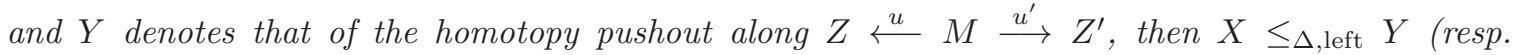
$X \leq_{\Delta+\text { nil,left }} Y$ ).

Remark 18. Note that the order $X \leq_{\Delta} Y$ is inverse to the order $M \leq_{\Delta} N$.

Remark 19. Note that Proposition [17] should be seen in the context of Bongartz [2, Lemma 1.1]. There it is shown that if

$$
0 \rightarrow M^{\prime} \rightarrow M \rightarrow M^{\prime \prime} \rightarrow 0
$$

is a short exact sequence, then $M$ degenerates to the pushout $N\lrcorner$ along an endomorphism of $M^{\prime}$, and $M$ degenerates to the pullback $N\left\ulcorner\right.$ along an endomorphism of $M^{\prime \prime}$.

Proof. (of Proposition [17). Note that $X$ and $Y$ are uniquely determined up to non-unique isomorphism. However, we have some freedom for the choice of the morphisms which complete $Z \stackrel{t}{\longleftarrow} N \stackrel{t^{\prime}}{\longrightarrow} Z^{\prime}$ and $Z \stackrel{u}{\longleftarrow} M \stackrel{u^{\prime}}{\longrightarrow} Z^{\prime}$ to the corresponding homotopy pushouts. We claim that we can choose the morphisms $w: Z \longrightarrow Y$ and $w^{\prime}: Z^{\prime} \longrightarrow Y$ so that the two squares and the patching of them in the following commutative diagram are homotopy cartesian:

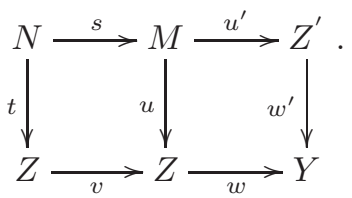

Indeed, by the statement of the proposition, the left square is homotopy cartesian and now we can form the homotopy pushout of $Z \stackrel{t}{\longleftarrow} N \stackrel{u^{\prime} s}{\longrightarrow} Z^{\prime}$, completing with morphisms $Z \stackrel{\eta}{\longrightarrow} W \stackrel{\rho}{\longleftarrow} Z^{\prime}$ such that we get the following homotopy cartesian square

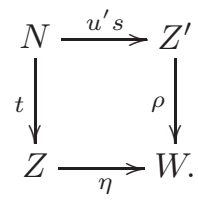

Since we have an equality $\eta t=\rho u^{\prime} s$, by properties of homotopy pushouts, we get a not necessarily unique morphism $\varphi: Z \longrightarrow W$ such that $\varphi v=\eta$ and $\varphi u=\rho u^{\prime}$. This leads to the following commutative diagram, where the left square and the patching of the two squares are homotopy cartesian:

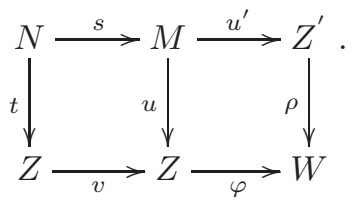


By [17, Lemma 9], one can replace $\varphi$ by an appropriate substitute so that the right square is also homotopy cartesian. But then $W$ is isomorphic to $Y$ and, looking at this isomorphism as an identification, we can choose $w^{\prime}=\rho$ and $w$ to be the mentioned substitute of $\varphi$.

Now the different homotopy cartesian squares fit as faces of the following almost-cube:

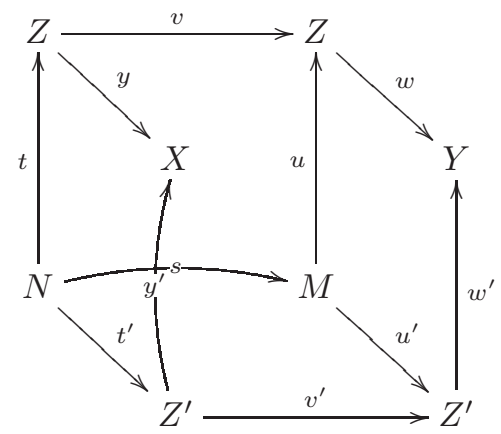

Since $X$ is a homotopy pushout, and since

$$
w v t=w u s=w^{\prime} u^{\prime} s=w^{\prime} v^{\prime} t^{\prime}
$$

there is a morphism $X \stackrel{d}{\longrightarrow} Y$ such that

$$
y d=w v \text { and } d y^{\prime}=w^{\prime} v^{\prime} .
$$

We can complete the vertical morphisms to distinguished triangles and obtain hence the commutative diagram with vertical sequences being distinguished triangles.

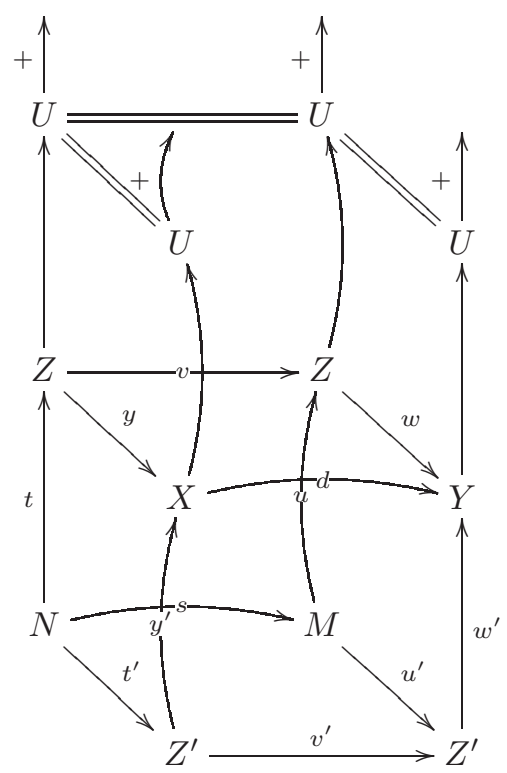

In particular, we get the following commutative diagram:

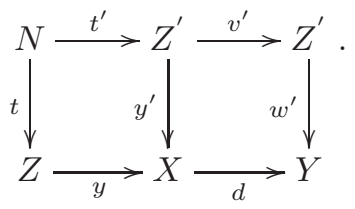

Its left square is homotopy cartesian by hypothesis and the patching of the two square is the pathching of the two squares in the diagram $\left(^{*}\right)$ above, and then is also homotopy cartesian. By [17. Lemma 9] again, replacing $d$ by a suitable $d^{\prime}$, we can assume without loss of generality that the right square is also homotopy cartesian. But this shows that $X \leq_{\Delta \text {,left }} Y$ (resp. $X \leq_{\Delta+\text { nil,left }} Y$ when $v$ and $v^{\prime}$ are nilpotent). 
Corollary 20. Suppose that $\mathcal{T}$ has split idempotents and is either the category of compact objects in an algebraic compactly generated triangulated category, or else has artinian endomorphism rings of objects. In the notation of Proposition [17, then

$$
M \leq_{\Delta+\text { nil }} N \Rightarrow X \leq_{\Delta+\text { nil }} Y \text {. }
$$

Proof. Indeed, using [17, we see that $X \leq_{\Delta+\text { nil,right }} Y \Leftrightarrow X \leq_{\Delta+\text { nil,left }} Y$ under these hypotheses.

\section{Degeneration of Zero and the zero objects in the Grothendieck Group}

Recall that, given full subcategories $\mathcal{U}$ and $\mathcal{V}$ of a triangulated category $\mathcal{T}$, then the subcategory $\mathcal{U} \star \mathcal{V}$ is the full subcategory of $\mathcal{T}$ consisting of the objects $M$ that fit in a distinguished triangle $U \longrightarrow M \longrightarrow V \longrightarrow U[1]$, with $U \in \mathcal{U}$ and $V \in \mathcal{V}$. It is well-known that the operation $\star$ is associative, in the sense that $(\mathcal{U} \star \mathcal{V}) \star \mathcal{W}=\mathcal{U} \star(\mathcal{V} \star \mathcal{W})$, for all subcategories $\mathcal{U}, \mathcal{V}, \mathcal{W}$ of $\mathcal{T}$ (see 1, Lemme 1.3.10]). If one puts $\mathcal{U}^{\star n}=\underbrace{\mathcal{U} \star \cdots \star \mathcal{U}}_{n \text { factors }}$, for each $n \geq 0$ (with the convention that $\mathcal{U}^{\star 0}=0$ ),

then $\mathcal{U}^{\text {ext }}=\bigcup_{n \in \mathbf{N}} \mathcal{U}^{\star n}$ is the extension closure of $\mathcal{U}$, that is, the smallest subcategory of $\mathcal{T}$ closed under extensions that contains $\mathcal{U}$. The smallest triangulated subcategory of $\mathcal{T}$ that contains $\mathcal{U}$, denoted $\operatorname{tria} \mathcal{T}(\mathcal{U})$, is

$$
\operatorname{tria} \mathcal{T}(\mathcal{U})=\bigcup_{n \in \mathbb{N}} \bigcup_{\left(r_{1}, \ldots, r_{n}\right) \in \mathbb{Z}^{n}} \mathcal{U}\left[r_{1}\right] \star \cdots \star \mathcal{U}\left[r_{n}\right]
$$

In other words, the objects of $\operatorname{tria}_{\mathcal{T}}(\mathcal{U})$ are precisely those $M$ admitting a sequence

$$
0=M_{0} \stackrel{f_{1}}{\longrightarrow} M_{1} \stackrel{f_{2}}{\longrightarrow} \cdots \stackrel{f_{n-1}}{\longrightarrow} M_{n-1} \stackrel{f_{n}}{\longrightarrow} M_{n}=M,
$$

where cone $\left(f_{k}\right)$ is isomorphic to $U_{k}\left[r_{k}\right]$, for some $U_{k} \in \mathcal{U}$ and some $r_{k} \in \mathbb{Z}$, for all $k=1, \ldots, n$.

Let $\mathcal{T}$ be a skeletally small triangulated category. All throughout this section we will put

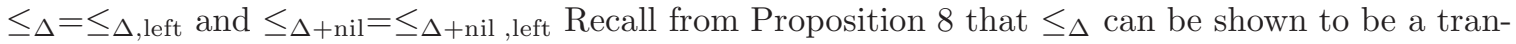
sitive relation in some cases. On the other hand, we will denote by $\preceq \Delta+$ nil the smallest transitive relation containing $\leq \Delta+$ nil.

Theorem 21. Let $\mathcal{S}$ be a set of objects in the triangulated category $\mathcal{T}$ such that $\mathcal{T}=\operatorname{tria}_{\mathcal{T}}(\mathcal{S})$, let $[\mathcal{S}]:=\{[S]: S \in \mathcal{S}\}$ denote the corresponding set of generators of the group $K_{0}(\mathcal{T})$ and let $\widehat{\mathcal{S}}$ be the subcategory of $\mathcal{T}$ consisting of the objects $X$ which are finite direct sums of shifts of objects in $\mathcal{S}$ and are such that $[X]=0$ in $K_{0}(\mathcal{T})$. Denote by

- $\mathcal{T}_{\Delta}^{0}$ (resp. $\mathcal{T}_{\Delta+\text { nil }}^{0}$ ) the full subcategory of $\mathcal{T}$ consisting of the objects $X$ such that $0 \leq_{\Delta} X$ (resp. $\left.0 \leq_{\Delta+\text { nil }} X\right)$

- and by $\mathcal{T}^{0}$ the (triangulated) subcategory of $\mathcal{T}$ consisting of the objects $M$ such that $[M]=0$ in the group $K_{0}(\mathcal{T})$.

Then the following assertions hold:

(1) An object $M$ is in $\mathcal{T}^{0}$ if, and only if, $M \leq_{\Delta} X$ (resp. $M \preceq \Delta+$ nil $X$ ), for some $X \in \widehat{\mathcal{S}}$. When $[\mathcal{S}]$ is a basis of $K_{0}(\mathcal{T})$ the objects $X$ can be chosen to be finite direct sums of shifts of objects in $\mathcal{\mathcal { S }}:=\{S \oplus S[2 k+1]: k \in \mathbb{Z} ; S \in \mathcal{S}\}$.

(2) $\mathcal{T}^{0}=\operatorname{tria}_{\mathcal{T}}\left(S \oplus S\left[t_{S}\right]: S \in \mathcal{S}\right)$, for every choice of odd integers $t_{S}$.

(3) $\mathcal{T}^{0}$ is the extension closure of $\mathcal{T}_{\Delta}^{0}$ (resp. $\mathcal{T}_{\Delta+\text { nil }}^{0}$ ).

Proof. (1) By Corollary 2, the 'if' part of this implication is clear. For the 'only if' part, we first claim that, for each $M \in \mathcal{T}$, one has that $M \preceq \Delta+$ nil $\oplus_{S \in \mathcal{S}} \oplus_{k \in \mathbb{Z}} S[k]^{m_{S, k}}$, where the $S$ are in $\mathcal{S}$ and the $m_{k, S}$ are nonnegative integers, all zero but a finite number. Recall that $\mathcal{T}=\operatorname{tria} \mathcal{T}(\mathcal{S})$, and so we have a finite sequence

$$
0=M_{0} \stackrel{f_{1}}{\longrightarrow} M_{1} \stackrel{f_{2}}{\longrightarrow} \cdots \stackrel{f_{n}}{\longrightarrow} M_{n}=M
$$

such that $C_{k}:=\operatorname{cone}\left(f_{k}\right)$ is a shift of some object of $\mathcal{S}$, for each $k=1, \ldots, n$. We will settle our claim by induction on $n>0$, the case $n=1$ being clear. Suppose now that $n>0$ and consider the induced triangle

$$
M_{n-1} \stackrel{f_{n}}{\longrightarrow} M \stackrel{g}{\longrightarrow} C_{n} \longrightarrow M_{n-1}[1]
$$


where $C_{n} \cong S[k]$, for some $S \in \mathcal{S}$ and $k \in \mathbb{Z}$. Taking the homotopy pushout of $f_{n}$ and the zero endomorphism $M_{n-1} \stackrel{0}{\longrightarrow} M_{n-1}$, we readily see that we have a distinguished triangle

$$
M_{n-1} \stackrel{\left(\begin{array}{c}
0 \\
f_{n}
\end{array}\right)}{\longrightarrow} M_{n-1} \oplus M \longrightarrow M_{n-1} \oplus C_{n} \longrightarrow M_{n-1}[1] .
$$

That is, we have $M \preceq \Delta+$ nil $M_{n-1} \oplus C_{n} \cong M_{n-1} \oplus S[k]$. The result then follows by induction since $A_{i} \preceq_{\Delta+\text { nil }} B_{i}$, for $i=1,2$, implies that $A_{1} \oplus A_{2} \preceq_{\Delta+\text { nil }} B_{1} \oplus B_{2}$.

We also claim that $M \leq_{\Delta} \oplus_{S \in \mathcal{S}} \oplus_{k \in \mathbb{Z}} S[k]^{m_{S, k}}$, for $S_{k}$ and $m_{S, k}$ as in the previous paragraph. Using again the sequence $(*)$ and bearing in mind that each cone $C_{k}$ is a shift of some object in $\mathcal{S}$, we consider the distinguished triangles

$$
M_{k-1} \stackrel{f_{k}}{\longrightarrow} M_{k} \longrightarrow C_{k} \longrightarrow M_{k-1}[1]
$$

for all $k \in\{1, \ldots, n-1\}$. Taking the direct sum of these distinguished triangles we get a distinguished triangle

$$
\left(\bigoplus_{k=1}^{n-1} M_{k}\right) \stackrel{\oplus_{k=1}^{n} f_{k}}{\longrightarrow}\left(M \oplus \bigoplus_{k=1}^{n-1} M_{k}\right) \longrightarrow\left(\bigoplus_{k=1}^{n} C_{k}\right) \longrightarrow\left(\bigoplus_{k=1}^{n-1} M_{k}\right)[1]
$$

and hence $M \leq_{\Delta} \bigoplus_{k=1}^{n} C_{k}$, as desired.

The last two paragraphs show that we have $M \preceq_{\Delta+\text { nil }} X$ and $M \leq_{\Delta} Y$, for objects $X, Y$ which are direct sums of shift of objects of $\mathcal{S}$. When in addition $M \in \mathcal{T}^{0}$, by Corollary 2 , we also have $[X]=[Y]=0$ in $K_{0}(\mathcal{T})$. Therefore we have that $X, Y \in \hat{\mathcal{S}}$. This proves assertion 1, except for the final statement.

To prove that final statement, suppose that $[\mathcal{S}]$ is a basis of $K_{0}(\mathcal{T})$. We shall prove that in this case each object of $\hat{\mathcal{S}}$ is a direct sum of objects of the form $S[k] \oplus S[l]=(S \oplus S[l-k])[k]$, with $S \in \mathcal{S}$ and exactly one of $l$ and $m$ being odd. This will end the proof. Let then take $X \in \hat{\mathcal{S}}$ and decompose it as $X=\oplus_{S \in \mathcal{S}} \oplus_{k \in \mathbb{Z}} S[k]^{m_{S, k}}$. Note that, due to the fact that $[\mathcal{S}]$ is a basis of $K_{0}(\mathcal{T})$, the summand $X_{S}=\oplus_{k \in \mathbb{Z}} S[k]^{m_{k, S}}$ also satisfies that $\left[X_{S}\right]=0$ in $K_{0}(\mathcal{T})$, for each $S \in \mathcal{S}$. So it is not restrictive to assume that $X=S\left[k_{1}\right]^{m_{1}} \oplus S\left[k_{2}\right]^{m_{2}} \oplus \cdots \oplus S\left[k_{r}\right]^{m_{r}}$, for some pairwise different integers $k_{1}, \ldots, k_{r}$, where, for simplicity, we have put $m_{k_{i}, S}=m_{i}>0$ for $i=1, \ldots, r$. We can reorder the summands in this last direct sum, so that $k_{i}$ is even, for $1 \leq i \leq t$, and $k_{i}$ is odd, for $t<i \leq n$. Bearing in mind that $[S[k]]=(-1)^{k}[S]$ in $K_{0}(\mathcal{T})$, that $[\mathcal{S}]$ is a basis of $K_{0}(\mathcal{T})$ and that $[X]=0$ in this latter abelian group, we conclude that $\sum_{i=1}^{t} m_{i}=\sum_{i=t+1}^{n} m_{i}$. We call $m(X)$ this last integer which is strictly positive when $X \neq 0$.

We now prove the result by induction on $m(X)>0$. If $m(X)=1$ then we have that $X \cong S[k] \oplus S[l]$, where $k$ is even and $l$ is odd, and we are done. If $m>1$, we put $q=\min \left\{m_{1}, \ldots, m_{r}\right\}$. We decompose $m_{1}=q+m_{1}^{\prime}$ and $m_{n}=q+m_{n}^{\prime}$ and $m_{i}^{\prime}=m_{i}$, for all $i \neq 1, n$. Then we have a decomposition $X=\left(S\left[k_{1}\right] \oplus S\left[k_{n}\right]\right)^{q} \oplus X^{\prime}$, where $k_{1}$ and $k_{n}$ are even and odd, respectively, and $X^{\prime}=\oplus_{i=1}^{n} S\left[k_{i}\right]^{m_{i}^{\prime}}$ is either zero or a nonzero summand such that $\left[X^{\prime}\right]=0$ in $K_{0}(\mathcal{T})$ and $m\left(X^{\prime}\right)<m(X)$. Then the induction hypothesis applies.

(2) Let $\left(t_{S}\right)_{S \in \mathcal{S}}$ be a collection of odd integers and put $\mathcal{D}:=\operatorname{tria} \mathcal{T}\left(S \oplus S\left[t_{S}\right]: S \in \mathcal{T}_{S}\right)$. It follows that each object of $\mathcal{S}$ is a direct summand of an object of $\mathcal{D}$ and since each object $T$ of $\mathcal{T}=\operatorname{tria} \mathcal{T}(\mathcal{S})$ is a finite iterated extension of objects of the form $S[k]$, with $S \in \mathcal{S}$ and $k \in \mathbb{Z}$, it easily follows that each such $T$ is a direct summand of an object of $\mathcal{D}$. This means that $\mathcal{D}$ is a dense triangulated subcategory of $\mathcal{T}$ in the terminology of [18]. Moreover, we clearly have $\mathcal{D} \subseteq \mathcal{T}^{0}$. But [18, Theorem 2.1] gives an order-preserving bijection between the dense triangulated subcategories of $\mathcal{T}$ and the subgroups of $K_{0}(\mathcal{T})$. Since $\mathcal{T}^{0}$ corresponds to 0 by this bijection we get that $\mathcal{D}=\mathcal{T}^{0}$, as desired.

(3) Note that assertion 2 implies assertion 3. Indeed, by the comments preceding Theorem 21, assertion 2 says that $\mathcal{T}^{0}$ is the extension closure of $\left\{\left(S \oplus S\left[t_{S}\right]\right)[n]: S \in \mathcal{S}\right.$ and $\left.n \in \mathbb{Z}\right\}$, for any choice of odd integers $t_{S}(S \in \mathcal{S})$. We may choose $t_{S}=1$ for each $S$, and then it is obvious that $0 \leq \Delta+$ nil $S \oplus S[1]$. Since we have inclusions

$$
\{(S \oplus S[1]): S \in \mathcal{S}\} \subset \mathcal{T}_{\Delta+\text { nil }}^{0} \subset \mathcal{T}_{\Delta}^{0} \subseteq \mathcal{T}^{0}
$$

assertion 3 immediately follows. 
Example 22. The following are examples of a triangulated category $\mathcal{T}$ and a set $\mathcal{S}$ of its objects that satisfy the hypotheses of Theorem 21 and, in addition, $[\mathcal{S}]$ is a basis of $K_{0}(\mathcal{T})$. Here $K$ is a commutative ring

(1) Call a dg $K$-algebra $A$ homologically non positive when $H^{k} A=0$, for all $k>0$, and call it homologically finite dimensional when $H^{*}(A)=\oplus_{k \in \mathbb{Z}} H^{k}(A)$ is a $K$-module of finite length. For instance, any Artin algebra is homologically non positive and homologically finite dimensional over its center, when viewed as $\operatorname{dg}$ algebra. Let $A$ be a homologically non positive homologically finite dimensional $\mathrm{dg}$ algebra and let $\mathcal{T}=\mathcal{D}_{f l}^{b}(A)$ be the subcategory of the derived category $\mathcal{D}(A)$ consisting of the $\operatorname{dg} A$-modules $M$ such that $H^{*}(M)=\oplus_{k \in \mathbb{Z}} H^{k}(M)$ has finite length as a $K$-module. When choosing as $\mathcal{S}$ a set of representatives, up to isomorphism in $\mathcal{D}(A)$, of the dg $A$-modules $S$ such that $H^{*}(S)=H^{0}(S)$ (i.e. its homology is concentrated in degree zero) and $H^{0}(S)$ is a simple $H^{0}(A)$-module, one has that $\mathcal{T}$ and $\mathcal{S}$ satisfy the hypotheses of Theorem 21 and $[\mathcal{S}]$ is a basis of $K_{0}(\mathcal{T})$. In particular, taking $A$ to be an Artin algebra, $\mathcal{T}=\mathcal{D}^{b}(\bmod -A)$ and $\mathcal{S}$ be a set of representatives, up to isomorphism, of the simple $A$-modules (viewed as stalk complexes in degree zero), the hypotheses of Theorem 21 hold and $[\mathcal{S}]$ is a basis of $K_{0}(\mathcal{T})$.

(2) Suppose that $\mathcal{A}$ is an additive category with a set $\mathcal{S}^{\prime}$ of objects such that $\mathcal{A}=\operatorname{add}\left(\mathcal{S}^{\prime}\right)$ and the Grothendieck group $K_{0}(\mathcal{A})$ is free with $\left\{[S]: S \in \mathcal{S}^{\prime}\right\}$ as a basis. Then the bounded homotopy category $\mathcal{T}=\mathcal{K}^{b}(\mathcal{A})$ and the set $\mathcal{S}=\mathcal{S}^{\prime}[0]$ of stalk complexes $S^{\prime}[0]$, with $S^{\prime} \in \mathcal{S}^{\prime}$, satisfy the hypotheses of Theorem 21 (see [15, Theorems 1.1 and 1.2]). This includes the case when $\mathcal{T}=\mathcal{K}^{b}(A-$ proj), where $A$ is a principal ideal domain or a semiperfect ring, in particular an Artin algebra, by taking as $\mathcal{S}^{\prime}$ the set of (isomorphism classes of) indecomposable projective $A$-modules

Lemma 23. Let $A$ be an Artin algebra and $S$ be a simple $A$-module. For each integer $k \neq-1,0$, the complex $M=S \oplus S[2 k+1]$ has the property that $[M]=0$ in $K_{0}\left(D^{b}(A-\bmod )\right)$, but it is not a $\Delta$-degeneration of zero: $0 \not \mathbb{\Delta}_{\Delta} M$.

Proof. Note that the homology module $H^{i}(M)$ is zero, except for $i=0$ and $i=-2 k-1$. If there is a distinguished triangle

$$
Z \stackrel{f}{\longrightarrow} Z \longrightarrow M \longrightarrow Z[1]
$$

in $D^{b}(A-\bmod )$, the associated sequence of homologies gives an exact sequence

$$
0 \longrightarrow H^{0}(Z) \stackrel{H^{0}(f)}{\longrightarrow} H^{0}(Z) \longrightarrow S \longrightarrow H^{1}(Z) \stackrel{H^{1}(f)}{\longrightarrow} H^{1}(Z) \longrightarrow 0,
$$

which forces $H^{0}(f)$ and $H^{1}(f)$ to be isomorphisms since they are a monomorphic and an epimorphic endomorphism, respectively, of finite length modules. Therefore $S=0$, and we get a contradiction.

Remark 24. Using Theorem 6 and Proposition 8 we see that in the situation of Lemma 23 the relations " $\leq_{\Delta+\text { nil }}$, " $\leq_{\Delta}$ ", and " $\leq_{\text {cdeg }}$ " coincide.

Recall that, for a skeletally small triangulated category $\mathcal{T}$, we denote by $\preceq \Delta+$ nil the smallest transitive relation on the set of isomorphism classes of objects in $\mathcal{T}$ containing $\leq_{\Delta+\text { nil }}$.

Proposition 25. Let $\mathcal{A}$ be any skeletally small abelian category for which $\mathcal{D}^{b}(\mathcal{A})$ is well-defined, i.e. it has Hom sets as opposed to proper classes, and let us identify $\mathcal{A}$ with the subcategory of $\mathcal{D}^{b}(\mathcal{A})$ consisting of objects $X$ such that $H^{i}(X)=0$, for $i \neq 0$. The following assertions hold:

(1) If $Y \leq_{\Delta+\text { nil }} X$ in $\mathcal{D}^{b}(\mathcal{A})$ and $X \in \mathcal{A}$, then $Y \in \mathcal{A}$.

(2) If there is an object in $\mathcal{A}$ which has a monomorphic endomorphism which is not an isomorphism, then there is an object $X$ in $\mathcal{T}:=\mathcal{D}^{b}(\mathcal{A})$ such that $0 \leq_{\Delta} X$ (and hence $[X]=0$ in $K_{0}(\mathcal{T})$ ), but $0 \npreceq \Delta+$ nil $X$.

Assertion 2 includes in particular the case of the category $\mathcal{A}=R-$ mod of finitely generated $R$-modules, when $K$ is a integral domain and $R$ is a noetherian $K$-algebra which is torsion-free as a $K$-module and does not contain the field of fractions of $K$ as a subalgebra. 
Proof. (1) Let us consider a distinguished triangle

$$
W \stackrel{\left(\begin{array}{c}
v \\
\alpha
\end{array}\right)}{\longrightarrow} W \oplus Y \longrightarrow X \longrightarrow W[1]
$$

in $D^{b}(\mathcal{A})$, where $v$ is a nilpotent endomorphism of $W$ and $X \in \mathcal{A}$. The long exact sequence of homologies gives that

$$
H^{j}(W) \stackrel{\left(\begin{array}{l}
H^{j}(v) \\
H^{j}(\alpha)
\end{array}\right)}{\longrightarrow} H^{j}(W) \oplus H^{j}(Y)
$$

is an isomorphism, for $j \neq 0,1$, and there is an exact sequence

$$
0 \longrightarrow H^{0}(W) \stackrel{\left(\begin{array}{l}
H^{0}(v) \\
H^{0}(\alpha)
\end{array}\right)}{\longrightarrow} H^{0}(W) \oplus H^{0}(Y) \longrightarrow X \longrightarrow H^{1}(W) \stackrel{\left(\begin{array}{l}
H^{1}(v) \\
H^{1}(\alpha)
\end{array}\right)}{\longrightarrow} H^{1}(W) \oplus H^{1}(Y) \longrightarrow 0
$$

in $\mathcal{A}$. Proving that $Y$ has homology concentrated in zero degree reduces to prove that if $\left(\begin{array}{c}w \\ g\end{array}\right): A \longrightarrow$ $A \oplus B$ is an epimorphism in $\mathcal{A}$, for some objects $A, B \in \mathcal{A}$, where $w$ is a nilpotent endomorphism of $A$, then $A=B=0$. This is clear when $w=0$. But if $w \neq 0$ and $m$ is the nilpotent index of $w$ (i.e. $\left.w^{m}=0 \neq w^{m-1}\right)$, then the composition

$$
A \stackrel{\left(\begin{array}{c}
w \\
g
\end{array}\right)}{\longrightarrow} A \oplus B \stackrel{\left(\begin{array}{ll}
w^{m-1} & 0
\end{array}\right)}{\longrightarrow} A
$$

is the zero map, which implies that $w^{m-1}=0$, thus yielding a contradiction.

(2) Let $f: Z \longrightarrow Z$ be a monomorphic endomorphism which is not an isomorphism and put $X=\operatorname{coker}(f)$. We then have an induced distinguished triangle

$$
Z \stackrel{f}{\longrightarrow} Z \longrightarrow X \longrightarrow Z[1]
$$

in $\mathcal{D}^{b}(\mathcal{A})$, thus showing that $0 \leq_{\Delta} X=X[0]$ in the later triangulated category. Suppose now that $0 \preceq_{\Delta+\text { nil }} X$. Then we have a sequence $0=X_{0}, X_{1}, \ldots, X_{n}=X$ in $D^{b}(\mathcal{A})$ such that $X_{i-1} \leq_{\Delta+\text { nil }} X_{i}$ and $X_{i} \neq 0$ for $i=1, \ldots, n$. By assertion 1 , we know that all $X_{i}$ are in $\mathcal{A}$. Replacing $X$ by $X_{1}$ if necessary, we get an object $X \neq 0$ of $\mathcal{A}$ such that $0 \leq_{\Delta+\text { nil }} X$ in $\mathcal{D}^{b}(\mathcal{A})$. We can fix a distinguished triangle

$$
Q \stackrel{u}{\longrightarrow} Q \longrightarrow X \longrightarrow Q[1]
$$

in $\mathcal{D}^{b}(\mathcal{A})$, where $u$ is a nilpotent endomorphism of $Q$. The long exact sequence of homologies gives then an exact sequence

$$
0 \longrightarrow H^{0}(Q) \stackrel{H^{0}(u)}{\longrightarrow} H^{0}(Q) \longrightarrow X \longrightarrow H^{1}(Q) \stackrel{H^{1}(u)}{\longrightarrow} H^{1}(Q) \longrightarrow 0
$$

in $\mathcal{A}$. But it is obvious that a nilpotent endomorphism of an object $A^{\prime} \in \mathcal{A}$ can be a monomorphism or an epimorphism only in case $A^{\prime}=0$. We then get $H^{j}(Q)=0$ for $j=0,1$, which in turn implies $X=0$ and hence a contradiction.

Finally, suppose now that $R$ - mod is the category of finitely generated modules over a noetherian $K$-algebra $R$ as indicated in the last statement of the proposition. Since the field of fractions of $K$ is not a subring of $R$, there must be a $\lambda \in K \backslash\{0\}$ such that $\lambda R \subsetneq R$. Due to the torsion-free condition of $R$ as a $K$-module, multiplication by $\lambda$ gives a monomorphism $u=u_{\lambda}: R \longrightarrow R$ in $R-\bmod$ which is not an epimorphism.

Example 26. Note that Proposition 25 shows that the stalk complex $\mathbb{Z} / p \mathbb{Z}$ in $\mathcal{D}^{b}(\mathbb{Z}$ - mod) has zero image in the Grothendieck group of the derived category and that $0 \npreceq \Delta+$ nil $\mathbb{Z} / p \mathbb{Z}$.

Remark 27. Proposition 25 shows that being degeneration of zero is strictly stronger than having zero image in the Grothendieck group. 


\section{REFERENCES}

[1] Alexander A. Beilinson, Joseph Bernstein, Pierre Deligne, Faisceaux pervers. Astérisque 100, vol. 1. Société Mathématique de France (1982).

[2] Klaus Bongartz, On Degenerations and Extensions of Finite Dimensional Modules, Advances of Mathematics 121 (1996) 245-287.

[3] Florian Eisele, The p-adic group ring of $S L_{2}\left(p^{f}\right)$, Journal of Algebra 410 (2014) 421-459.

[4] Florian Eisele, Blocks with a generalized quaternion defect group and three simple modules over a 2-adic ring, Journal of Algebra 456 (2016) 294-322.

[5] Pierre Gabriel, Finite representation type is open, Proceedings of the International Conference on Representations of Algebras (Carleton University, Ottawa, Ontario, 1974), Paper No. 10, 23 pp. Carleton Mathematics Lecture Notes, No. 9, Carleton University, Ottawa, Ontario, 1974

[6] Naoya Hiramatsu, Degenerations of graded Cohen-Macaulauy modules, Journal of Commuatative algebra 7 (2015) 221-239.

[7] Naoya Hiramatsu, On the stable hom relation and stable degenerations of Cohen-Macaulay modules, Journal of Pure and Applied Algebra 222 (2018) 2609-2625.

[8] Bernt Tore Jensen, Xiuping Su and Alexander Zimmermann, Degeneration for derived categories, Journal of Pure and Applied Algebra 198 (2005) 281-295.

[9] Bernt Tore Jensen, Xiuping Su and Alexander Zimmermann, Degeneration-like orders for triangulated categories, Journal of Algebra and Applications 4 (2005) 587-597.

[10] Berhnard Keller, Dong Yang, and Guodong Zhou, The Hall algebra of a spherical object, Journal of the London Mathematical Society 80 (2009) 771-784.

[11] Bernhard Keller and Sarah Scherotzke, Graded quiver varieties and derived categories, Journal für die reine und angewandte Mathematik $\mathbf{7 1 3}$ (2016) 85-127.

[12] Henning Krause, Cohomological Length Functions, Nagoya Math. Journal 223 (2016) 136-161.

[13] Amnon Neeman, Triangulated Categories, Princeton University Press, Princeton MA 2001

[14] Christine Riedtmann, Degenerations for representations of quivers with relations, Annales Scientifiques de l'École Normale Supérieure 19 (1986) 275-301.

[15] David E.V. Rose A note on the Grothendieck group of an additive category. Vestnik Chelyabinskogo Gosudarstvennogo Universiteta. Matematika, Mekhanika, Informatika. Nauchny Zhurnal no 317 (2015) 135-139. Available at https://arxiv.org/pdf/1109.2040.pdf

[16] Manuel Saorín and Alexander Zimmermann, An Axiomatic Approach for Degenerations in Triangulated Categories, Applied Categorical Structures 24 (2016) no 4, 385-405.

[17] Manuel Saorín and Alexander Zimmermann, Symmetry of the definition of degeneration in triangulated categories, Algebr Represent Theory (2018), https://doi.org/10.1007/s10468-018-9799-z.

[18] Robert W. Thomason, The classification of triangulated subcategories. Compositio Mathematica 105 (1997), 1-27.

[19] Yuji Yoshino, On degeneration of modules, Journal of Algebra 278 (2004) 217-226.

[20] Yuji Yoshino, Stable degeneration of Cohen-Macaulay modules, Journal of Algebra 332 (2011) 500-521.

[21] Zhengfang Wang, Triangle order $\leq_{\Delta}$ in Singular Categories, Algebras and Representation Theory 19 (2016) 397-404.

[22] Grzegorz Zwara, A degeneration-like order for modules, Archiv der Mathematik 71 (1998) 437-444.

[23] Grzegorz Zwara, Degenerations of finite dimensional modules are given by extensions, Compositio Mathematica 121 (2000) 205-218.

Departemento de Matemáticas,

Universidad de Murcia, Aptdo. 4021

30100 Espinardo, Murcia,

SPAIN

E-mail address: msaorinc@um.es

Université de Picardie,

DÉPARTEMENT DE Mathématiques et LAMFA (UMR 7352 DU CNRS),

33 RUE St LEU,

F-80039 Amiens Cedex 1,

FRANCE

E-mail address: alexander.zimmermann@u-picardie.fr 